\title{
The Application of Specific Nursing in Perioperative Patients of Thoracic Surgery and Postoperative Recovery and Improvement of Patients' Negative Emotions
}

\author{
Fen Xue ${ }^{1}$, Junrong Ding², Min Lu ${ }^{3 *}$, Yanjun $\mathrm{Mao}^{3 *}$ \\ ${ }^{1}$ Special Needs Ward I, Shanghai Pulmonary Hospital, Shanghai, China \\ ${ }^{2}$ Department of Thoracic Surgery, Shanghai Pulmonary Hospital, Shanghai, China \\ ${ }^{3}$ Nursing Department, Shanghai Pulmonary Hospital, Shanghai, China \\ Email: *maqeu0@163.com, *mkquo8@163.com
}

How to cite this paper: Xue, F., Ding, J.R., Lu, M. and Mao, Y.J. (2021) The Application of Specific Nursing in Perioperative Patients of Thoracic Surgery and Postoperative Recovery and Improvement of Patients' Negative Emotions. Journal of Biosciences and Medicines, 9, 169-181.

https://doi.org/10.4236/jbm.2021.96016

Received: May 10, 2021

Accepted: June 22, 2021

Published: June 25, 2021

\begin{abstract}
Objective: To study the application of specific nursing in perioperative patients of thoracic surgery and postoperative recovery and improvement of patients' negative emotions. Patients and Methods: A total of 88 patients in the thoracic surgery operating room of our hospital were selected and divided into group A (specific nursing, $n=44$ ) and group $B$ (routine nursing, $n=44$ ). The anxiety and depression status of the two groups were assessed by the Self-rating Anxiety Scale (SAS) and the Self-rating Depression Scale (SDS). The relevant respiratory parameters after surgery, including total lung capacity, maximum respiration flow-rate, tidal volume, and vital capacity were observed. The extubation time, ICU hospitalization time, and VAS scores of postoperative pain were compared, with a score of 10 being full. The higher the score, the higher the pain. The incidence of postoperative wound infection, pulmonary infection, respiratory tract infection and the satisfaction with the operation were compared. Sf-36 scale was used to score the physical function, life function, psychological function and quality of life of the patients in the two groups. Results: The surgical effect of group A was significantly higher than that of group B (all $p<0.05$ ). The total lung capacity, maximum respiration flow-rate, tidal volume and vital capacity of group A were significantly higher than those of group B (all $p<0.05$ ). The extubation time and hospitalization time of group A were significantly higher than those of group $\mathrm{B}$ (all $p<0.05$ ). VAS score of group A was significantly lower than that group $\mathrm{B}$ (all $p<0.05$ ). The infection rate of group $\mathrm{A}$ was significantly lower than that in group B (all $p<0.05)$. Conclusion: Specific nursing has higher
\end{abstract}


application value than routine nursing, which is worth further promotion in clinic.

\section{Keywords}

Specific Nursing, Thoracic Surgery, Postoperative Recovery, Negative Emotions

\section{Introduction}

Most patients of thoracic surgery have complicated conditions, difficult treatment, high surgical risk, large surgical wounds and easy infection, which will affect their postoperative life and require long-term self-cultivation and recovery [1]. For the long-term inconvenience of life and trauma and pain, it will seriously affect the psychological mood of patients and easily bring an invisible psychological pressure and anxiety to patients, which will cause panic to patients [2]. For complicated operations in this situation, routine postoperative nursing has become increasingly unable to meet the needs of patients. Routine nursing only observes the basic index of the patient's body and uses the same nursing model for the general public. It is difficult to detect the potential needs of patients. Routine nursing does not establish a good doctor-patient relationship with patients. Patients' psychological problems are difficult to be detected by nursing staff, which may cause patients' emotional problems, then affect surgical treatment and increase patients' pain. However, with the development of the times and the improvement of medical technology, the demand for patients has become more and more important. Therefore, a kind of special nursing based on routine nursing has been developed in clinic, which can pay attention to the quality of life of patients, take care of patients' psychological emotions and provide meticulous care for patients' physical conditions. We call it specific nursing [3]. The so-called specific nursing is to develop a new type of care model that is devoted to satisfying patients according to different needs of different patients. Specific nursing runs through the whole process of surgical treatment until patients were cured and discharged.

This research is to study the application of specific nursing in perioperative patients of thoracic surgery and postoperative recovery and improvement of patients' negative emotions.

\section{Materials and Methods}

\subsection{Baseline Data}

A total of 88 patients in the operating room of thoracic surgery in our hospital were selected as research objects and divided into two groups, A and B, with 44 cases in each group. In group A, patients were cared by specific nursing, and there were 24 males and 20 females. The average age was $(45.34 \pm 7.03)$ years old. BMI was $(25.41 \pm 2.92) \mathrm{kg} / \mathrm{m}^{2}$. In group $\mathrm{B}$, patients were cared by routine 
nursing, and there were 32 males and 12 females. The average age was $(44.98 \pm$ $7.98)$ years old. BMI was $(26.35 \pm 2.24) \mathrm{kg} / \mathrm{m}^{2}$.

\subsection{Exclusion and Inclusion Criteria}

Inclusion criteria: The patient was diagnosed as requiring thoracic surgery and had complete clinical data. They were between 20 and 60 years old, with good life characteristic and no psychosis. In this study, patients and their families were informed and signed the consent form. This study was approved by the hospital ethics committee.

Exclusion criteria: Patients who did not cooperate, patients with operation contraindication, hepatic and kidney function obstacle and cogulation disturbance were excluded. Pregnant or lactating women, patients with disturbance of consciousness and patients who hide something to medical staff were excluded.

\subsection{Nursing Methods}

In group A, patients were cared by specific nursing. Specific measures are as follows:

1) Psychological nursing for patients: Patients will produce stress and anxiety before and after surgery. Nursing staff often have to communicate with patients to relieve anxiety. They can relieve patients' negative emotions and find out their psychological problems by explaining medical knowledge and successful cases. They guide patients' bad emotions through the key issues and encourage patients to improve their self-confidence. Nursing staff should also adopt the opinions of patients, learn about the deficiencies, amend, strengthening and continue to provide personalized care with high quality and high service for patients.

2) Health education for patients: Before carrying out health education, nursing staff should communicate with patients to understand their demands and answer their questions. According to patients' cognitive level of disease and surgery, the personalized plan is formulated to meet patients' knowledge needs and let patients have a further understanding of surgical treatment and postoperative recovery. In this process, nurses must constantly communicate with patients and observe their conditions. This will make the operation easier to conduct. Meanwhile, the occurrence of postoperative complications can be prevented, which can be treated early.

3) Life nursing for patients: Nursing staff need to provide a comfortable environment for patients to stay in the hospital. They need to clean and disinfect the wards every day to prevent the growing of bacteria. Nursing staff need to open the window for a few hours a day to keep the air flowing every day, so that patients can breathe fresh air and keep patients happy.

4) Nutrition supplement for patients: According to different stages of patients' postoperative recovery, different feeding plans should be made. At the beginning of the operation, patients need to choose flowing habits' food and bland diet that 
is easy to digest and absorb. They need to tell patients about food taboos, which avoid eating spicy food, beef, mutton and other stimulating food, so as to avoid recurrence and infection of wound. One to two days after surgery, patients can eat some nourishing food, such as chicken soup, fish soup and other food. And some safe food with high protein and fiber can be appropriately selected according to the recovery of patients.

5) Complications care for patients: nursing staff must adjust the temperature and humidity of the ward reasonably and observe the patient's physical indicators and changes in vital signs every day to prevent infection or complications. Ask the patient if there is any discomfort and contact the doctor as soon as possible so as not to delay treatment. Nursing staff should instruct patients to pay attention to their own hygiene conditions and to expel sputum as soon as possible. If the sputum is difficult to discharge, patients need to receive atomization inhalation therapy, two to three times a day. Patients should be instructed to get out of bed, walk more in the ward or outdoors, and practice deep breathing. Patients should be instructed to cough and expectorate correctly to prevent atelectasis, and nursing staff should pat patients on the back to help them discharge sputum when they cough. Also regularly observe the drainage situation and squeeze the drainage tube, so as not to occur flow tube blockage. Changes in drainage fluid color and drainage volume should also be observed frequently to analyze for infection or complications. If there is any abnormality, you should inform the doctor in time and take corresponding measures.

6) Pain nursing for patients: Patients' will feel pain more and more obvious after operation. Nursing staff need to timely pay attention to and learn about patient needs. For a small degree of pain, the nursing staff need to tell the patient how to relieve the pain and help patients carry out it. If there is a high degree of pain, it is necessary to contact the doctor in time, inform the doctor about the details of the patient and provide a basis for the doctor's treatment.

7) The guidance of discharge for patients: When the patient is cured and leave hospital, the nursing staff will help the patient technically handle the relevant discharge procedures and leave the contact information of the patient, so as to pay attention to the physical condition of the patient after discharge. And they will inform patients to notice announcements and the time of return visit and provide convenience for the health recovery of patients.

In group $\mathrm{B}$, patients were cared by routine nursing. To observe the change of patients disease. To provide diet and the guidance of activities. To inform announcements to patients and their families. To aware of relevant issues, communicate with the doctor about the patient's condition and solve it in time.

\subsection{Observation Indexes}

1) To make a questionnaire that marked effect, effective and ineffective of patients after operation, then compared the results.

2) The anxiety and depression status of patients were assessed by the Self- 
rating Anxiety Scale (SAS) [4] and the Self-rating Depression Scale (SDS) [5]. The higher the score, the more serious of patient's anxiety and depression. The critical value of SAS is 50 and the critical value of SDS score is 53 .

3) Comparison of relevant respiratory parameters, including vital capacity, maximum respiration flow-rate, tidal volume and vital capacity of patients in two groups after operation.

4) Comparison of extubation time, ICU hospitalization time and postoperative pain of two groups and score by Visual Analogue Scale/Score (VAS) [6], with 0 - 10 scores. The higher the score, the higher the pain.

5) Comparison of the incidence of incision infection after operation, pulmonary infection, respiratory tract infection of two groups.

6) Comparison of the results of surgical satisfaction (satisfactory, rather satisfactory, basically satisfactory and dissatisfied) of patients in two groups.

7) Sf-36 scale [7] was used to score the physical function, life function, psychological function and quality of life of the patients in the two groups. The full score was 100, and the higher the score, the higher the quality of life.

\subsection{Statistical Methods}

SPSS20.0 (SPSS, Inc., Chicago, IL, USA) was used for statistical analysis. T test was used for measurement data and expressed as mean number \pm standard deviation $(\mathrm{x} \pm \mathrm{s})$. The enumeration data were tested by chi-square test and expressed by percentage [n (\%)]. The difference was statistically significant with $p<0.05$.

\section{Results}

\subsection{Baseline Data}

There was no significant difference in baseline data of gender, age, BMI, smoking history, drinking history and obesity status between the two groups (all $p>$ 0.05). More details are shown in Table 1.

\subsection{Comparison of Efficacy of Patients between the Two Groups after Operation}

In group $\mathrm{A}$, there were 32 cases with marked effect, 10 cases with effective effect and 2 cases with ineffective effect, with an effective rate of $95.45 \%$. In group B, there was 9 cases with marked effect, 21 cases with effective effect and 14 cases with ineffective effect, with an effective rate of $68.18 \%$. The difference was statistically significant of patients in two groups (all $p<0.05$ ). More details are shown in Table 2.

\subsection{Comparison of SAS and SDS Scores before and after Nursing between the Two Groups}

There was no significant difference in SAS scores and SDS scores between group $A$ and group B before nursing (all $p>0.05$ ). After nursing, SAS and SDS scores of group A and group B decreased significantly compared with those before 
Table 1. The baseline data of patients between the two groups.

\begin{tabular}{ccccc}
\hline Classification & Group A $(\mathrm{n}=44)$ & Group B $(\mathrm{n}=44)$ & $\mathrm{t} / \chi^{2}$ value & $p$ value \\
\hline Gender & & & 3.143 & 0.076 \\
Male & $24(54.55)$ & $32(72.73)$ & & \\
Female & $20(45.45)$ & $12(27.27)$ & & 0.409 \\
Age (years old) & $45.34 \pm 7.03$ & $44.98 \pm 7.98$ & 0.224 & 0.085 \\
BMI (kg/m $\left.{ }^{2}\right)$ & $25.41 \pm 2.92$ & $26.35 \pm 2.24$ & 1.694 & 0.133 \\
Smoking history & & & & \\
Yes & $16(36.36)$ & $23(52.27)$ & 2.256 & \\
No & $28(63.63)$ & $21(47.73)$ & & 0.820 \\
Drinking history & & & & \\
Yes & $30(68.18)$ & $29(65.91)$ & 0.051 & \\
No & $14(31.82)$ & $15(34.09)$ & & 0.466 \\
Obesity status & & & & \\
Yes & $34(77.27)$ & $31(70.45)$ & 0.529 & \\
No & $10(23.73)$ & $13(29.55)$ & & \\
\hline
\end{tabular}

Table 2. Comparison of efficacy of patients between the two groups after operation.

\begin{tabular}{cccccc}
\hline Grouping & $\mathrm{n}$ & Marked effect & Effective & Ineffective & Effective rate \\
\hline Group A & 44 & $32(72.73)$ & $10(22.73)$ & $2(4.55)$ & $42(95.45)$ \\
Group B & 44 & $9(20.45)$ & $21(47.73)$ & $14(31.82)$ & $30(68.18)$ \\
$\mathrm{X}^{2}$ & - & - & - & 11.00 \\
$p$ & & - & - & - & $<0.001$ \\
\hline
\end{tabular}

nursing (all $p<0.05$ ). After nursing, SAS and SDS scores in group A were significantly decreased compared with those in group B (all $p<0.05$ ). More details are shown in Figure 1 and Figure 2.

\subsection{Comparison of Respiratory Parameters of Patients after Operation between the Two Groups}

The total lung capacity, maximum respiration flow-rate, tidal volume, vital capacity of groups A was $(5.36 \pm 1.98) \mathrm{L},(4.99 \pm 0.47) \mathrm{L} / \mathrm{S},(0.56 \pm 0.12) \mathrm{L},(5.27 \pm$ 1.37) L, respectively. The total lung capacity, maximum respiration flow-rate, tidal volume, vital capacity of groups B was $(4.47 \pm 1.26) \mathrm{L}$, $(4.39 \pm 1.19) \mathrm{L} / \mathrm{S}$, $(0.38 \pm 0.09) \mathrm{L},(4.67 \pm 1.14) \mathrm{L}$, respectively. Group A was significantly higher than group B. The difference was statistically significant (all $p<0.05$ ). More details are shown in Table 3.

\subsection{Comparison of Extubation Time, ICU Hospitalization Time, VAS Score between the Two Groups}

The extubation time, ICU hospitalization time, VAS score of group A was (5.13 


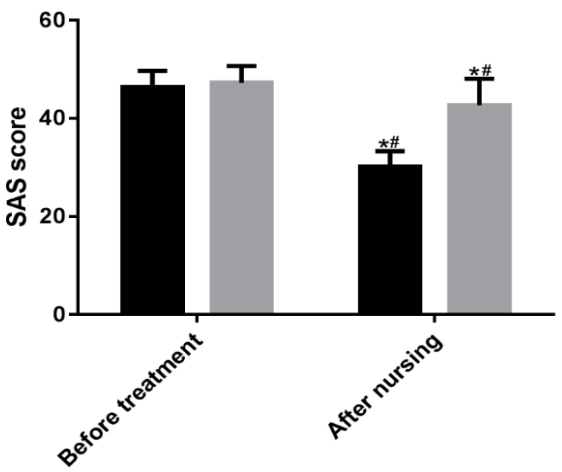

In SAS scores, there was no significant difference between the two groups before treatment (all $p>$ $0.05)$. After treatment, group A was significantly lower than group B (all $p<0.05)$. There was a significant decrease in the two groups after treatment (all $p<0.05$ ). Notice: ${ }^{*}$ indicates the difference within the group after nursing and before nursing (all $p<0.05$ ) and "indicates the difference between the group (all $p<0.05$ ).

Figure 1. Comparison of SAS scores of patients before and after operation between the two groups.

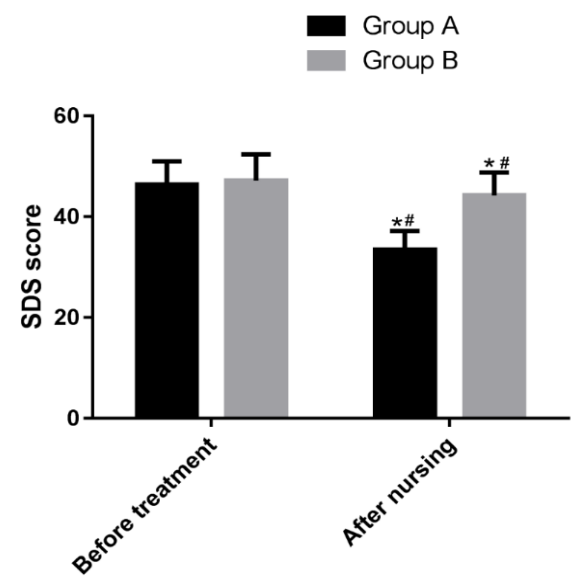

In SDS scores, there was no significant difference between the two groups before treatment (all $p>$ 0.05). After treatment, group A was significantly lower than group B (all $p<0.05$ ). There was a significant decrease in the two groups after treatment (all $p<0.05$ ). Notice: ${ }^{*}$ indicates the difference within the group after nursing and before nursing (all $p<0.05)$ and "indicates the difference between the group (all $p<0.05$ ).

Figure 2. Comparison of SDS scores of patients before and after operation between the two groups.

Table 3. Comparison of respiratory parameters of patients after operation between the two groups.

\begin{tabular}{ccccc}
\hline Respiratory parameters & Group A $(\mathrm{n}=44)$ & Group B (n=44) & t & $\boldsymbol{p}$ \\
\hline Total lung capacity (L) & $5.36 \pm 1.98$ & $4.47 \pm 1.26$ & 2.515 & 0.013 \\
Maximum respiration flow-rate (L/S) & $4.99 \pm 0.47$ & $4.39 \pm 1.19$ & 3.111 & 0.002 \\
Tidal volume (L) & $0.56 \pm 0.12$ & $0.38 \pm 0.09$ & 7.960 & $<0.001$ \\
Vital capacity (L) & $5.27 \pm 1.37$ & $4.67 \pm 1.14$ & 2.233 & 0.028 \\
\hline
\end{tabular}


$\pm 1.03) \mathrm{d},(5.33 \pm 1.29) \mathrm{d},(3.28 \pm 1.25)$, respectively. The extubation time, ICU hospitalization time, VAS score of group B was $(6.24 \pm 1.35) \mathrm{d},(8.44 \pm 2.11) \mathrm{d}$, $(6.48 \pm 1.67)$, respectively. All the items in group B were significantly higher than those in group A. The difference was statistically significant (all $p<0.05$ ). More details are shown in Table 4.

\subsection{Comparison of the Incidence of Infection between the Two Groups after Operation}

In group $A$, there was 1 case with wound infection, 3 cases with pulmonary infection, 2 cases with respiratory tract infection. The total infection rate was $13.64 \%$. In group $\mathrm{B}$, there were 8 cases with wound infection, 13 cases with pulmonary infection, 10 cases with respiratory tract infection. The total infection rate was $70.45 \%$. The infection rate of group B was significantly higher than that in group A. The difference was statistically significant (all $p<0.05$ ). More details are shown in Table 5.

\subsection{Comparison of Satisfaction with the Operation of Patients between the Two Groups}

In group A, there were 37 patients with satisfaction, 4 patients with rather satisfaction, 2 patients with basic satisfaction and 1 patient with dissatisfaction. The total satisfaction rate was $97.73 \%$. In group $\mathrm{B}$, there were 20 patients with satisfaction, 9 patients with rather satisfaction, 7 patients with basic satisfaction and 8 patients with dissatisfaction. The total satisfaction rate was $81.82 \%$. The total satisfaction rate of group A was significantly higher than taht in group B. The difference was statistically significant (all $p<0.05$ ). More details are shown in Table 6.

Table 4. Comparison of extubation time, ICU hospitalization time, VAS score between the two groups

\begin{tabular}{cccc}
\hline Grouping & Extubation time (d) & $\begin{array}{c}\text { ICU hospitalization } \\
\text { time (d) }\end{array}$ & VAS score \\
\hline Group A & $5.13 \pm 1.03$ & $5.33 \pm 1.29$ & $3.28 \pm 1.25$ \\
Group B & $6.24 \pm 1.35$ & $8.44 \pm 2.11$ & $6.48 \pm 1.67$ \\
$\mathrm{t}$ & 4.336 & 8.342 & 10.18 \\
$p$ & $<0.001$ & $<0.001$ & $<0.001$ \\
\hline
\end{tabular}

Table 5. Comparison of the incidence of infection between the two groups after operation.

\begin{tabular}{cccccc}
\hline Grouping & Cases & $\begin{array}{c}\text { Wound } \\
\text { infection }\end{array}$ & $\begin{array}{c}\text { Pulmonary } \\
\text { infection }\end{array}$ & $\begin{array}{c}\text { Respiratory tract } \\
\text { infection }\end{array}$ & Infection rate \\
\hline Group A & 44 & $1(2.27)$ & $3(6.82)$ & $2(4.55)$ & $6(13.64)$ \\
Group B & 44 & $8(18.18)$ & $13(29.55)$ & $10(22.73)$ & $31(70.45)$ \\
$\mathrm{X}^{2}$ & & 6.065 & 7.639 & 6.175 & 29.151 \\
$p$ & & 0.013 & 0.005 & 0.013 & $<0.001$ \\
\hline
\end{tabular}


Table 6. Comparison of satisfaction with the operation of patients between the two groups.

\begin{tabular}{ccccccc}
\hline Grouping & $\mathbf{n}$ & Satisfactory & $\begin{array}{c}\text { Rather } \\
\text { satisfactory }\end{array}$ & $\begin{array}{c}\text { Basically } \\
\text { satisfaction }\end{array}$ & Dissatisfied & $\begin{array}{c}\text { Total } \\
\text { satisfaction } \\
\text { degree }\end{array}$ \\
\hline Group A & 44 & $37(84.09)$ & $4(9.09)$ & $2(4.55)$ & $1(2.27)$ & $43(97.73)$ \\
Group B & 44 & $20(45.45)$ & $9(20.45)$ & $7(15.91)$ & $8(18.18)$ & $36(81.82)$ \\
$\mathrm{X}^{2}$ & & 15.861 & 2.256 & 3.094 & 6.065 & 6.065 \\
$p$ & $<0.001$ & 0.133 & 0.078 & 0.013 & 0.013 \\
\hline
\end{tabular}

Table 7. Comparison of quality of life scores between the two groups.

\begin{tabular}{cccccc}
\hline Grouping & Cases & Physical function & Life function & $\begin{array}{c}\text { Psychological } \\
\text { function }\end{array}$ & Quality of life \\
\hline Group A & 44 & $88.35 \pm 15.27$ & $92.37 \pm 10.72$ & $89.72 \pm 8.18$ & $93.12 \pm 9.26$ \\
Group B & 44 & $67.83 \pm 13.28$ & $80.17 \pm 12.47$ & $79.38 \pm 9.27$ & $60.29 \pm 10.78$ \\
t & & 6.726 & 4.921 & 5.548 & 15.32 \\
$p$ & & 0.001 & $<0.001$ & $<0.001$ & $<0.001$ \\
\hline
\end{tabular}

\subsection{Comparison of Quality of Life Scores of Patients between the Two Groups}

In group $\mathrm{A}$, the scores of physical function, life function, psychological function, quality of life was (88.35 \pm 15.27$),(92.37 \pm 10.72),(89.72 \pm 8.18),(93.12 \pm 9.26)$, respectively. In group $\mathrm{B}$, the scores of physical function, life function, psychological function, quality of life was $(67.83 \pm 13.28),(80.17 \pm 12.47),(79.38 \pm$ 9.27), (60.29 \pm 10.78$)$, respectively. The scores of group A were significantly higher than those in group B. The difference was statistically significant (all $p<0.05$ ). More details are shown in Table 7.

\section{Discussion}

Although thoracic surgery is common in clinical operation, surgical treatment is still more complex, difficult [8] and painful [9] [10]. Long-term surgical treatment will cause harmful effects to patients [11], which will also bring anxiety and pressure to patients. Patient's ignorance of the operation may cause patients to resist the operation, which may affect the progress of operation. This will consume amount of human and time resources.

The difference of patients and disease with surgery will produce the different related problems with following the surgery. Routine nursing can no longer meet the needs of different patients. Routine nursing is the use of the same methods of care for patients with different types of surgery. This nursing methods are difficult to adapt to all patients and they have poor flexibility. Although routine nursing can save costs, the uniformity of routine care is not easy to find the changes and characteristics of the patient's disease. If the nursing effect is not good, it is easy to delay the treatment of the disease. 
So there is a new model of specific nursing in the clinic. Specific nursing is the improvement of routine nursing and comprehensive nursing of patients is carried out with patients as the center [12]. In this way, patients' wrong perceptions can be changed to medical staff. It can reduce the conflict of doctor-patient relationship, improve the satisfaction of patients [13] and reduce the psychological burden of patients. Therefore, the improvement of the clinical efficacy can help patients heal from hospital as soon as possible.

Negative emotions can affect physical health [14] and vice versa. Long-term wound pain and patients' own worry about disease treatment can easily cause psychological burden on patients. Patients are also experiencing surgical, pipeline and other stimulation factors. Patients may suffer from insomnia, loss of appetite, depression of mood, anxiety and other emotions, which may affect the treatment process of the disease and affect the physical recovery. Therefore, psychological guidance is an important part of specific nursing. Only when the patient is in a good mood can he have a good effect on promoting recovery [15] and improve the treatment rate of the disease. In this study, we compared SAS and SDS scores before and after nursing in group A with specific nursing and group B patients with routine nursing. The results showed that the SAS and SDS scores of the two groups were significantly decreased after the nursing. But the SAS and SDS scores of group A decreased more than those of group B. The difference was statistically significant. This shows that emotional interference can improve the psychological distress and negative impact of patients in specific nursing [16]. We also compared postoperative efficacy between group A and group B. The results showed that the effective rate of group A was significantly higher than that of group B. It shows that specific nursing has a good stimulative effect on surgical treatment. LaganaAS et al. [17] studied anxiety and depression in endometrial patients, and the results showed that psychological factors play an important role in determining the severity of symptoms and the effectiveness of treatment. We can further speculate that comprehensive emotional care for patients is beneficial to treatment in specific nursing, which is similar to the results in this thesis.

Airway secretions are usually blocked in the respiratory tract after long-term thoracic surgery. If it is not cleaned up in time, it will affect the patient's breathing and cause dyspnea. If the patient does not expectorate in time, it will increase the probability of pulmonary infection in patients [18]. In this thesis, we compared respiratory parameters and infection rate of patients between two groups after operation. The results showed that the total lung capacity, maximum respiration flow-rate, tidal volume, vital capacity of groups A were significantly higher than those in group B. The infection rate of group B was significantly higher than group A. The difference was statistically significant. It can be shown that the nursing intervention of sputum excretion and promoting sputum to patients in specific nursing can improve the respiratory function of patients. It can shorten the recovery progress and help patients. In ad- 
dition, specific nursing can effectively reduce the occurrence of postoperative infection and complications by paying close attention to patients' in various conditions. Anderson E A [19] et al. showed that the incidence of postoperative hypertension can be reduced by making patients feel good or reducing preoperative anxiety. It can be shown that the occurrence of postoperative complications can be reduced by intervening patients' emotions in specific nursing. It is similar to the results in this thesis.

During specific nursing, we intervened physical health, mental emotion, nutrition supplement, physical pain and postoperative rehabilitation of patients. It can effectively improve the patient's treatment confidence, improve the operation efficiency, shorten the operation time, and be beneficial to the postoperative recovery of patients. In this study, we compared extubation time and ICU hospitalization time of patients between two groups. The results showed that the extubation time and ICU hospitalization time of groups A was significantly higher than group B. The difference was statistically significant. The results confirmed this conclusion. Specific nursing runs through the whole process of surgery. Nursing staff will pay close attention to patients' pain and timely find problems and contact doctors to take measures to effectively reduce the pain degree of patients. In this study, VAS scores were performed on patients. The results showed that the VAS score of groups A was significantly lower than group B. The difference was statistically significant. During specific nursing, nursing staff make personalized nutritious food according to patients' physical needs and dietary preferences, which can enhance patients' immunity and reduce postoperative infection rate and the occurrence of complications. In this study, the incidence of infection of groups A was significantly lower than group B. The difference was statistically significant. The results confirmed this conclusion. Eckard C [20] et al. reduced pain symptoms of patients by using intelligence and the Internet to divert their attention. It indicates that it is beneficial to patients' pain symptoms by emotional guidance. It is similar to the results in this thesis.

In this thesis, we also compared satisfaction and quality of life of patients. The results showed that the satisfaction of groups A was significantly higher than group B. The difference was statistically significant. The results showed that the physical function, life function, psychological function, quality of life of groups A was significantly higher than group B. The difference was statistically significant. It indicates that specific nursing is more valuable in application than routine nursing [21].

\section{Conclusion}

To sum up, the implementation of specific nursing can effectively improve the treatment efficiency rate, reduce the time of staying in hospital, the incidence of pain and infection of patients, improve the satisfaction of patients with surgery and improve the quality of life of patients after surgery. In short, specific nursing is more valuable in application than routine nursing. 


\section{Authors' Contributions}

FX designed the study and drafted the manuscript. JD and ML were responsible for the collection and analysis of the experimental data. YM revised the manuscript critically for important intellectual content. All authors read and approved the final manuscript.

\section{Conflicts of Interest}

The authors declare no conflicts of interest regarding the publication of this paper.

\section{References}

[1] D’Agostino, R.S., Jacobs, J.P., Badhwar, V., et al. (2017) Department of Thoracic Surgery Adult Cardiac Surgery Database: 2017 Results and Quality Update. Breast Surgery Annual Report, 103, 18-24. https://doi.org/10.1016/j.athoracsur.2016.11.001

[2] Svensson, M., Nilsson, U. and Svantesson, M. (2016) Patient Experience While Waiting for Day Surgery. Journal of Clinical Nursing, 25, 2600-2608. https://doi.org/10.1111/jocn.13304

[3] McAlister, F.A., Laupacis, A. and Armstrong, P.W. (2017) Find the Right Balance between Precision Medicine and Personalized Care. CMAJ, 189, E1065-E1068. https://doi.org/10.1503/cmaj.170107

[4] Svanborg, P. and Åsberg, M. (1994) A New Self-Rating Scale Based on the Comprehensive Psychopathological Rating Scale for Depression and Anxiety. Acta Psychiatrica Scandinavica, 89, 21-28. https://doi.org/10.1111/j.1600-0447.1994.tb01480.x

[5] Zung, W.W. K. (1965) Self-Reported Depression Scale. General Psychiatry Archives, 12, 63-70. https://doi.org/10.1001/archpsyc.1965.01720310065008

[6] Bijur, P.E., Silver, W. and Gallagher, E. J. (2001) Reliability of Visual Analogue Scales for Measuring Acute Pain. Academic Emergency Medicine, 8, 1153-1157. https://doi.org/10.1111/j.1553-2712.2001.tb01132.x

[7] Ware Jr. J.E. and Sherbourne, C.D. (1992) MOS 36 Short-Term Health Survey (SF-36), I. Conceptual Framework and Project Selection. Medical, 473-483. https://doi.org/10.1097/00005650-199206000-00002

[8] Shahian, D.M., O’Brien, S.M, Filardo, G., et al. (2009) Cardiac Surgery Risk Model of the Thoracic Surgery Society 2008: Part 1-Coronary Artery Bypass Grafting. Yearbook of Thoracic Surgery, 88, S2-S22. https://doi.org/10.1016/j.athoracsur.2009.05.053

[9] Katz, J., Jackson, M., Kavanagh, B.P., et al. (1996) Acute Pain after Thoracic Surgery Can Predict Long-Term Pain after Thoracotomy. Journal of Clinical Pain, 12, 50-55. https://doi.org/10.1097/00002508-199603000-00009

[10] Kalso, E., Perttunen, K. and Kaasinen, S. (1992) Thoracic Surgery Pain. Acta Anaesthesiologica Scandinavica, 36, 96-100. https://doi.org/10.1111/j.1399-6576.1992.tb03430.x

[11] Bayliff, C.D., Massel, D.R., Inculet, R.I., et al. (1999) Propranolol Prevents Arrhythmia after General Thoracic Surgery. The Annals of Thoracic Surgery, 67, 182-186. https://doi.org/10.1016/S0003-4975(98)01226-0

[12] Han, C.J. (2016) Conceptual Analysis of Personalized Nursing Care. Nursing Forum, 51, 32-39. https://doi.org/10.1111/nuf.12117 
[13] Stratton, L., Dannewitz, H., Margrett, J., et al. (2018) The Difference between Direct Nursing Professional Burn and Personalized Nursing in Long-Term Care Environment. Aging Innovation, 2, 457. https://doi.org/10.1093/geroni/igy023.1713

[14] Rosenthal, L., Earnshaw, V.A., Carroll-Scott, A., et al. (2015) Bullying Based on Weight and Race: The Health Association of Urban Youth. Journal of Health Psychology, 20, 401-412. https://doi.org/10.1177/1359105313502567

[15] Powell, R., Scott, N.W., Manyande, A., et al. (2016) Adult Psychological Preparation and Postoperative Prognosis under General Anesthesia. Cochrane Systematic Evaluation Database, 5.

[16] Raz, D.J., Sun, V., Kim, J.Y., et al. (2016) Long-Term Effects of Interdisciplinary Supportive Care Interventions for Lung Cancer Survivors after Surgery. Yearbook of Thoracic Surgery, 101, 495-503. https://doi.org/10.1016/j.athoracsur.2015.07.031

[17] Laganà, A.S., LaRosa, V.L., Rapisarda, A.M.C., et al. (2017) Anxiety and Depression in Patients with Endometriosis: Effects and Management Challenges. International Journal of Women's Health, 9, 323-330. https://doi.org/10.2147/IJWH.S119729

[18] Fernández-Pérez, E.R., Keegan, M.T., Brown, D.R., et al. (2006) Intraoperative Tidal Volume as a Risk Factor for Respiratory Failure after Pneumonectomy. Anesthesiology: American Journal of Anesthesiologists, 105, 14-18. https://doi.org/10.1097/00000542-200607000-00007

[19] Anderson, E.A. (1987) Preoperative Preparation of Cardiac Surgery Helps to Restore, Reduce Psychological Distress and Reduce the Incidence of Acute Postoperative Hypertension. Journal of Counseling and Clinical Psychology, 55, 513. https://doi.org/10.1037/0022-006X.55.4.513

[20] Eckard, C., Asbury, C., Bolduc, B., et al. (2016) Integrating Technology into Treatment Options Can Help Alleviate Chronic Pain. Journal of Pain Management and Medicine, 2.

[21] Reuben, D.B. and Sinsky, C.A. (2018) From Trading Tasks to Personalized Care: A New Vision of the Doctor's Role. Family Medicine Yearbook, 16, 168-169. https://doi.org/10.1370/afm.2203 